\title{
Two-Step Spectral Clustering Controlled Islanding Algorithm
}

\author{
Lei Ding, Member, IEEE, Francisco M. Gonzalez-Longatt, Senior Member, IEEE, Peter Wall, and \\ Vladimir Terzija, Senior Member, IEEE
}

\begin{abstract}
Controlled islanding is an active and effective way of avoiding catastrophic wide area blackouts. It is usually considered as a constrained combinatorial optimization problem. However, the combinatorial explosion of the solution space that occurs for large power systems increases the complexity of solving it. This paper proposes a two-step controlled islanding algorithm that uses spectral clustering to find a suitable islanding solution for preventing the initiation of wide area blackouts by un-damped electromechanical oscillations. The objective function used in this controlled islanding algorithm is the minimal power-flow disruption. The sole constraint applied to this solution is related to generator coherency. In the first step of the algorithm, the generator nodes are grouped using normalized spectral clustering, based on their dynamic models, to produce groups of coherent generators. In the second step of the algorithm, the islanding solution that provides the minimum power-flow disruption while satisfying the constraint of coherent generator groups is determined by grouping all nodes using constrained spectral clustering. Simulation results, obtained using the IEEE 9-, 39-, and 118-bus test systems, show that the proposed algorithm is computationally efficient when solving the controlled islanding problem, particularly in the case of a large power system.
\end{abstract}

Index Terms-Constrained spectral clustering, controlled islanding, graph theory, normalized spectral clustering.

\section{INTRODUCTION}

$\mathbf{C}$ ONTROLLED islanding of a power system is an efficient corrective measure for limiting system blackouts after a large disturbance has occurred. It limits the occurrence and consequences of blackouts by splitting the power system into a group of smaller, islanded power systems, or islands. The essence of an islanding solution is determining a suitable set of transmission lines that need to be disconnected to create a set of electrically isolated islands. Controlled islanding can be used to cope with different power system extremes, such as un-damped

Manuscript received March 23, 2011; revised July 19, 2011, November 10, 2011, and February 19, 2012; accepted April 11, 2012. Date of publication June 06, 2012; date of current version January 17, 2013. This work was supported in part by the EPSRC under Grant EP/G055211/1, and in part by NSFC under Grant 51107071 and Shandong University project (IIFSDU 2010TB024). Paper No. TPWRS-00251-2011.

L. Ding is with the School of Electrical Engineering, Shandong University, Jinan 250061, China, and also with the School of Electrical and Electronic Engineering, The University of Manchester, Manchester M13 9PL, U.K. (e-mail: dinglei@sdu.edu.cn).

F. M. Gonzalez-Longatt, P. Wall, and V. Terzija are with the School of Electrical and Electronic Engineering, The University of Manchester, Manchester M13 9PL, U.K. (e-mail: terzija@ieee.org).

Color versions of one or more of the figures in this paper are available online at http://ieeexplore.ieee.org.

Digital Object Identifier 10.1109/TPWRS.2012.2197640 oscillations, voltage collapse, cascading trips, etc. This paper proposes an algorithm for determining suitable islanding solutions for the scenario of un-damped electromechanical oscillations that are not accompanied by voltage instability.

To create stable islands, the islanding solution must satisfy a large number of constraints, such as load-generation balance, generator coherency, availability of transmission lines, thermal limits, voltage stability, transient stability, etc. It would be too complicated to search for a solution satisfying all of these constraints or even confirm if such a solution exists. Considering only a sub-set of these constraints, such as load-generation balance and generator coherency, allows a set of feasible candidate islanding solutions to be produced. This set of candidates can be coordinated with other corrective measures to find a final islanding solution that satisfies all constraints [1]-[6]. This approximation reduces the complexity of the controlled islanding problem; this is particularly useful when dealing with large networks [1]-[4].

Existing methods in the literature for determining islanding solutions can be classified according to the objective function used. The two main types of objective function are minimal power imbalance and minimal power-flow disruption.

Methodologies for minimal power imbalance minimize the power imbalance within the islands formed to reduce the amount of load that must be shed after system splitting [1]-[4], [7]-[11]. Methodologies for finding islanding solutions with the minimal power-flow disruption minimize the change of the power flow pattern within the system following system splitting [12]-[14].

The difference between power imbalance and power-flow disruption is that the power imbalance can be expressed by the algebraic sum of active power (considering the direction of power flow) on each disconnected transmission line, while the power-flow disruption can be expressed by the arithmetical sum of active power on each disconnected transmission line.

In [1], a two-phase Ordered Binary Decision Diagram (OBDD) method based on a simplified graph is presented to find islands with low power imbalance that contain coherent generators. In [2], the Breadth First Search (BFS) and Depth First Search (DFS) algorithms are used to find the islanding solution that separates coherent generator groups, with minimal power imbalance. Other algorithms that can be used to find islands with the minimal power flow imbalance include Angle Modulated Particle Swarm Optimization [4], and the Krylov Subspace method [7].

Finding a solution with minimal power imbalance is an $N P$-hard problem and has been shown to be a special case of 
the 0-1 knapsack problem [1]. There is no known algorithm that can efficiently solve problems of this type within polynomial time [1], [9].

Most existing algorithms overcome this challenge by using heuristic search methods, or by only solving the problem for a simplified network model, or a select subset of the original power system [1]-[9]. For example, when using an OBDD based method in online applications, the network model should be simplified to contain less than approximately 40 nodes [3], [11].

Simplifying the network model reduces the solution space. It is possible that some of the solutions that are lost during the simplification will be better than the final solution found by the algorithm [11]. Heuristic search methods are usually quite flexible and have satisfactory computational efficiency. However, the solution quality cannot be guaranteed since these methods tend to converge to local, rather than global, minima.

Spectral partitioning and multi-level kernel $k$-means methods are proposed in [12] and [13] to find the islanding solution with the minimal power-flow disruption. Both methods have excellent computational efficiency, but do not consider generator coherency [15]. This neglect of generator behavior means that the stability of the islands produced cannot be guaranteed. In addition, direct application of spectral clustering without constraints often leads to a single node being separated from the rest of the graph [16]. The flaws in these two solution types are clearly unacceptable when attempting the controlled islanding of a power system.

In this paper, a novel two-step Spectral Clustering Controlled Islanding algorithm (the SCCI algorithm) will be presented. In the first step of the SCCI algorithm, the generator nodes are grouped using normalized spectral clustering. The results of this grouping serve as pair-wise constraints in the next step of the SCCI algorithm, in which every node is grouped based on constrained spectral clustering. This constrained spectral clustering uses power flow data to producing an islanding solution with minimal power-flow disruption. Therefore, the two-step SCCI algorithm proposed here can identify, in real time, an islanding solution that has minimal power-flow disruption and satisfies the constraint of generator coherency.

The main body of the paper is organized as follows. Section II introduces the controlled islanding problem and basic concepts of spectral clustering. In Section III, the execution of the proposed SCCI algorithm is discussed. In Section IV, the new algorithm is applied to the IEEE 9-bus, 39-bus, and 118-bus test systems to demonstrate its performance. Section V concludes the paper.

\section{Controlled Islanding and Spectral Clustering}

In this section, some basics concepts of graph theory are introduced. The controlled islanding problem is then defined as a constrained optimization problem that is converted into a graph-cut problem. A possible method for solving this type of problem, spectral clustering, is introduced.

\section{A. Graph Theory Preliminaries}

In graph theory, an undirected graph-model $\mathbf{G}\left(\mathbf{V}, \mathbf{V}_{\mathbf{G}}, \mathbf{E}, \mathbf{W}\right)$ can be used to describe an $m$-gen- erator and $n$-bus power system. In the above graph-model, the node set $\mathbf{V}=\left\{v_{1}, \ldots, v_{n}\right\}$ and the edge set $\mathbf{E}$, with elements $e_{i j}(i, j=1, \ldots, n)$, denote the buses and transmission lines, respectively. $\mathbf{V}_{\mathbf{G}}$ is a subset of the node set $\mathbf{V}$ that contains only those buses with generators directly connected to them. The matrix $\mathbf{W}$ is a set of edge weights.

For convenience, only the bisection case is presented in this paper. Bisection of a graph $\mathbf{G}$ splits it into two sub-graphs $\mathbf{G}_{1}\left(\mathbf{V}_{1}, \mathbf{V}_{\mathbf{G} 1}, \mathbf{E}_{1}, \mathbf{W}_{1}\right)$ and $\mathbf{G}_{2}\left(\mathbf{V}_{2}, \mathbf{V}_{\mathbf{G} 2}, \mathbf{E}_{2}, \mathbf{W}_{2}\right)$ by removing the edges connecting these two sub-graphs, with each sub-graph representing a sub-system of the original power system. Here, $\mathbf{V}_{\mathbf{1}}$ and $\mathbf{V}_{\mathbf{2}}$ are disjoint subsets of $\mathbf{V}$, i.e., $\mathbf{V}_{\mathbf{1}} \cap \mathbf{V}_{\mathbf{2}}=\varnothing$ and $\mathbf{V}_{\mathbf{1}} \cup \mathbf{V}_{\mathbf{2}}=\mathbf{V}$. In the same way, $\mathbf{V}_{\mathbf{G} \mathbf{1}}$ and $\mathbf{V}_{\mathbf{G}_{2}}$ are defined as two disjoint subsets of $\mathbf{V}_{\mathbf{G}}$, while they are also subsets of $\mathbf{V}_{\mathbf{1}}$ and $\mathbf{V}_{\mathbf{2}}$, respectively.

The set of edges removed to separate these sub-graphs is called the cutset. The sum of the weights of the edges within this cutset is called the cut, which is defined as [17]

$$
\operatorname{cut}\left(\mathbf{V}_{\mathbf{1}}, \mathbf{V}_{\mathbf{2}}\right)=\sum_{i \in \mathbf{V}_{\mathbf{1}}, j \in \mathbf{V}_{\mathbf{2}}} w_{i j} .
$$

The graph-cut problem is then defined as finding the cutset that bisects the graph with minimum cut [16]. It is common that the islanding solution, for a large power system, will require the system to be split into more than two islands; this is achieved using recursive bisection [16].

\section{B. Controlled Islanding}

The set of sub-graphs formed using the recursive bisection approach represent the islands that must be created to achieve controlled islanding. To ensure that stable islands are produced, the islands formed should have minimal power-flow disruption and satisfy the constraint of generator coherency, as discussed below.

1) Generator Coherency: A large disturbance in a power system can initiate un-damped electromechanical oscillations; these oscillations can cause generators to lose their coherency. To create stable islands, the generators within any island formed must be approximately synchronous.

Based on the classical linearized generator swing equation, with damping neglected, the linearized second-order dynamic model of an $m$-generator power system can be expressed in the following matrix form [18]:

$$
\ddot{\mathbf{x}}=\mathbf{A x}
$$

where $\mathbf{x}=\left[\Delta \delta_{1}, \ldots, \Delta \delta_{m}\right]^{T}$ and $\Delta \delta$ is the generator angle deviation from a steady state operating point $\delta_{0}$ and $\mathbf{A}$ is the system state matrix. According to the theory of slow coherency, separating the generators into two groups is equivalent to an arbitrary division of matrix $\mathbf{A}$ into two sub-matrices $\mathbf{A}_{\mathbf{1 1}}$ and $\mathbf{A}_{\mathbf{2 2}}$ that represent the sub-systems $\mathbf{G}_{\mathbf{1}}$ and $\mathbf{G}_{\mathbf{2}}$ (see Fig. 1) [19].

The sum of the Frobenius norms of the off-diagonal sub-matrices $\mathbf{A}_{\mathbf{1 2}}$ and $\mathbf{A}_{21}$ can be used to define the dynamic coupling $\boldsymbol{S}$ between subsystems $\mathbf{G}_{\mathbf{1}}$ and $\mathbf{G}_{\mathbf{2}}$ [19]:

$$
S=\left\|\mathbf{A}_{12}\right\|+\left\|\mathbf{A}_{21}\right\| .
$$




$$
\text { Subsystem } \mathbf{G}_{1} \underset{\mathbf{A}}{\longrightarrow}=\left[\begin{array}{c:c}
\mathbf{A}_{11} & \mathbf{A}_{12} \\
\hdashline \mathbf{A}_{21} & \mathbf{A}_{22}
\end{array}\right], \text { Subsystem } \mathbf{G}_{2}
$$

Fig. 1. Division of the system matrix $\mathbf{A}$ into two sub-matrices $\mathbf{A}_{11}$ and $\mathbf{A}_{22}$.

In this paper, the focus is solely upon the scenario of un-damped electromechanical oscillations that are not accompanied by voltage instability. In this scenario, if it is assumed that the reactive power balance can be controlled via local compensation, the effects of the reactive power and bus voltage magnitude can be neglected as they have a negligible impact on the dynamic coupling. Therefore, (3) can be rewritten as follows:

$$
S=\sum_{j \in \mathbf{V}_{\mathbf{G} 2}} \sum_{i \in \mathbf{V}_{\mathbf{G} 1}}\left(\frac{\partial P_{i j}}{\partial \delta_{i j}} \cdot\left(\frac{1}{H_{i}}+\frac{1}{H_{j}}\right)\right)
$$

where $\partial P_{i j} / \partial \delta_{i j}$ are the synchronizing coefficients and $H_{i}$ is the inertia constant of the $i$ th generator.

When exposed to electromechanical oscillations generators with strong dynamic coupling will swing together, whereas generators with weak dynamic coupling will swing against one another [18]. Therefore, the problem of finding coherent generator groups is equivalent to an optimization problem of finding the weakest dynamic coupling between different generator groups, as shown in (5):

$$
\begin{aligned}
& \min S \\
& =\min _{\mathbf{V}_{\mathbf{G} 1}, \mathbf{V}_{\mathbf{G} 2} \subset \mathbf{V}_{\mathbf{G}}}\left(\sum_{j \in \mathbf{V}_{\mathbf{G} \mathbf{2}}} \sum_{i \in \mathbf{V}_{\mathbf{G} 1}}\left(\frac{\partial P_{i j}}{\partial \delta_{i j}} \cdot\left(\frac{1}{H_{i}}+\frac{1}{H_{j}}\right)\right)\right) .
\end{aligned}
$$

2) Objective Function: Minimal power imbalance and minimal power-flow disruption, defined according to (6) and (7), respectively, can both be used as objective functions of controlled islanding. Each objective will produce a different solution with different advantages and disadvantages [3], [20]:

$$
\begin{array}{r}
\min _{\mathbf{V}_{\mathbf{1}}, \mathbf{V}_{\mathbf{2}} \subset \mathbf{V}}\left(\left|\sum_{i \in \mathbf{V}_{\mathbf{1}}, j \in \mathbf{V}_{\mathbf{2}}} P_{i j}\right|\right) \\
\min _{\mathbf{V}_{\mathbf{1}}, \mathbf{V}_{2} \subset \mathbf{V}}\left(\sum_{i \in \mathbf{V}_{\mathbf{1}}, j \in \mathbf{V}_{\mathbf{2}}}\left|P_{i j}\right|\right)
\end{array}
$$

where $P_{i j}$ denotes the value of the active power on the transmission line between node $i$ and $j$.

The use of minimal power imbalance as the objective function creates islands with a similar level of load and generation, i.e., a good load-generation balance. This property of the objective function minimizes the amount of load that must be shed following system splitting.

The use of minimal power-flow disruption as the objective function creates islands with the minimum change from the predisturbance power-flow pattern. This property of the objective function improves the transient stability of the islands, reduces the possibility of overloading the transmission lines within the island, and eases the islands eventual reintegration with the rest of the system [20].
When attempting to ensure stability after system splitting the transient stability, rather than load-generation balance, should be the primary concern because an island with a negative stability margin and good load-generation balance will collapse. However, an island with a positive stability margin and a poor load-generation balance can be stabilized through load shedding.

Based on the properties of these objective functions the minimal power flow disruption is used in this paper, it also has the additional benefit of reducing the complexity of the problem faced, the details of this reduction in complexity are described in Section IV-B.

Considering the complexity of large interconnected power systems, it might be that the consideration of active power flows only would not lead to the optimal splitting solution. The inclusion of some heuristic knowledge inherent for every single system, or additional assessment of reactive power flows and voltage stability related challenges would probably lead to a more efficient final splitting solution. However, the complexity of such an approach might be too high and even not practical enough. This should be addressed in future research projects.

3) Controlled Islanding Problem: The controlled islanding problem that is solved in this paper consists of a minimal power-flow disruption objective function (7) and a generator coherency constraint (5). In [21] some basic results in solving such an optimization problem are given.

These two optimization problems are combined to form the SCCI algorithm (8). This is done by first solving (5), to find a set of coherent generator groups, and then solving (7) subject to these generator groups:

$$
\begin{aligned}
& {\left[\mathbf{V}_{\mathbf{G} \mathbf{1}}^{\star}, \mathbf{V}_{\mathbf{G} \mathbf{2}}^{\star}\right]=} \\
& \underset{\mathbf{V}_{\mathbf{G} 1}, \mathbf{V}_{\mathbf{G} \mathbf{2}} \subset \mathbf{V}_{\mathbf{G}}}{\operatorname{argmin}}\left(\sum_{j \in \mathbf{V}_{\mathbf{G} \mathbf{2}}} \sum_{i \in \mathbf{V}_{\mathbf{G} \mathbf{1}}}\left(\frac{\partial P_{i j}}{\partial \delta_{i j}} \cdot\left(\frac{1}{H_{i}}+\frac{1}{H_{j}}\right)\right)\right) \\
& \min _{\mathbf{V}_{\mathbf{1}}, \mathbf{V}_{2} \subset \mathbf{V}}\left(\sum_{i \in \mathbf{V}_{1}, j \in \mathbf{V}_{\mathbf{2}}}\left|P_{i j}\right|\right) \\
& \text { subject to } \mathbf{V}_{\mathbf{G} \mathbf{1}}^{\star} \subset \mathbf{V}_{\mathbf{1}} \mathbf{V}_{\mathbf{G} \mathbf{2}}^{\star} \subset \mathbf{V}_{2} .
\end{aligned}
$$

Here, argmin stands for the argument of the minimum, i.e., $\left[\mathbf{V}_{\mathbf{G} \mathbf{1}}^{\star}, \mathbf{V}_{\mathbf{G} \mathbf{2}}^{\star}\right]$ is the node grouping that minimizes the objective function of (5) [22].

\section{Spectral Clustering}

Defining the edge weight of the graph using the synchronizing coefficient or the absolute active power on the transmission line allows the problem of finding the weakest dynamic coupling and the minimum power flow disruption, respectively, to be converted into graph-cut problems.

Spectral clustering is the tool used in this paper to solve these graph-cut problems. In this subsection, two types of spectral clustering will be introduced, namely un-normalized and normalized spectral clustering.

1) Un-Normalized Spectral Clustering: The theory behind un-normalized spectral clustering can be briefly described as follows. 
Un-Normalized Spectral Clustering clusters the nodes into two subsets based on the Laplacian Matrix $\mathbf{L}$, which is defined for a graph $\mathbf{G}$ as [16]

$$
\mathbf{L}=\mathbf{D}-\mathbf{W}
$$

where $\mathbf{D}$ is a diagonal degree matrix that contains diagonal elements $D_{i i}$ that are equal to the total weight of the edges connected to node $i$. Defined in this way, the edge weight matrix $\mathbf{W}$ and the Laplacian Matrix $\mathbf{L}$ are both symmetric for any undirected graph.

The un-normalized spectral clustering algorithm, for the case of bisection, can be executed using the following steps [16]:

1) Compute the first two eigenvectors $\vartheta_{1}, \vartheta_{2}$ of the Laplacian matrix $\mathbf{L}$.

2) Let $\mathbf{J} \in \mathbf{R}^{n \times 2}$ be the matrix containing the vectors $\vartheta_{1}, \vartheta_{2}$ as columns. Let $y_{i} \in \mathbf{R}^{2}$ be the vector corresponding to the $i$ th row of $\mathbf{J}$.

3) Cluster the nodes $y_{i} \in \mathbf{R}^{2}$ into clusters $c_{1}, c_{2}$ using a clustering algorithm, e.g., the $k$-medoids algorithm [23].

Unfortunately, the solution for bisecting the graph using un-normalized spectral clustering often consists of simply separating one node from the rest of the graph. This form of solution is clearly unacceptable for an islanding solution.

2) Normalized Spectral Clustering: Normalized spectral clustering uses the sum of the node weights within each sub-graph as a balancing condition, to prevent the application of spectral clustering from simply separating a single node. This gives rise to the concept of a normalized cut (Ncut) [17], which is, defined as

$$
\operatorname{Ncut}\left(\mathbf{V}_{\mathbf{1}}, \mathbf{V}_{\mathbf{2}}\right)=\frac{\operatorname{cut}\left(\mathbf{V}_{\mathbf{1}}, \mathbf{V}_{\mathbf{2}}\right)}{\operatorname{weig}\left(\mathbf{V}_{1}\right)}+\frac{\operatorname{cut}\left(\mathbf{V}_{\mathbf{1}}, \mathbf{V}_{\mathbf{2}}\right)}{\operatorname{weig}\left(\mathbf{V}_{2}\right)}
$$

where weig $\left(\mathbf{V}_{\mathbf{1}}\right)=\sum_{i \in \mathbf{V}_{\mathbf{1}}} D_{i}$ and is thus the total sum of the weights of the nodes in $\mathbf{G}_{\mathbf{1}}$. The weig $\left(\mathbf{V}_{\mathbf{2}}\right)$ is similarly defined for $\mathbf{G}_{\mathbf{2}}$. The inclusion of the node weights as a balancing condition acts to discourage the creation of a sub-graph with very low weight. The normalized spectral clustering method can be used to split the graph with minimum Ncut.

For the case of bisection, this can be achieved using the following steps [16], [17]:

1) Compute the first two eigenvectors $\vartheta_{1}, \vartheta_{2}$ of the generalized eigen-problem $\mathbf{L} \vartheta=\lambda \mathbf{D} \vartheta$.

2) Let $\mathbf{J} \in \mathbf{R}^{n \times 2}$ be the matrix containing the vectors $\vartheta_{1}, \vartheta_{2}$ as columns. Let $y_{i} \in \mathbf{R}^{2}$ be the vector corresponding to the $i$ th row of $\mathbf{J}$.

3) Cluster the nodes $y_{i} \in \mathbf{R}^{2}$ into clusters $c_{1}, c_{2}$ using a clustering algorithm, such as the k-medoids algorithm.

\section{Two-STEP SPeCtral Clustering CONTROLLED ISLANDING ALGORITHM}

In this section, the two-step SCCI algorithm, proposed for solving the optimization problem expressed in (8), is presented. Solving this optimization problem is equivalent to determining a suitable islanding solution.

This solution can be found by constructing two graphs, based on the objective function and constraint from (8), and applying the SCCI algorithm to find the minimum cut of these two graphs.

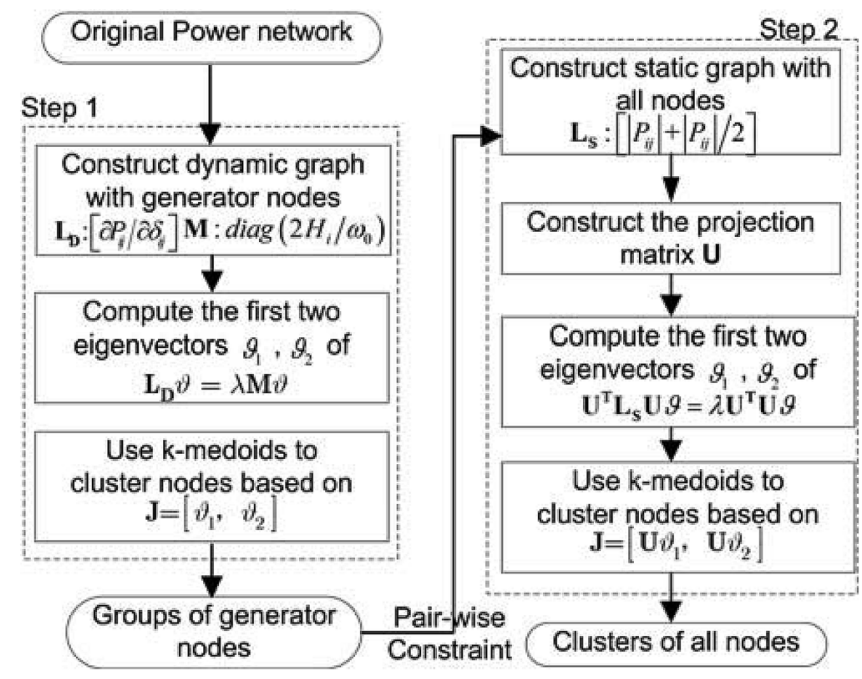

Fig. 2. Flowchart of the SCCI algorithm.

In the first step of the SCCI algorithm, the dynamic graph $\mathbf{G}_{\mathbf{D}}$ is constructed. It only contains the generator nodes and its edge weights of this graph are the synchronizing coefficients $\partial P_{i j} / \partial \delta_{i j}$ that describe the dynamic coupling between the nodes $i$ and $j$. To satisfy the generator coherency constraint (5), the generator nodes are grouped using the normalized spectral clustering algorithm, introduced in Section II-C2. These groups of generator nodes then serve as constraints for the second step of the SCCI algorithm.

In the second step of the algorithm, the static graph $\mathbf{G}_{\mathbf{S}}$ is constructed using power flow data. It contains every node and the edge weights are defined as the absolute value of the active power exchange between nodes $i$ and $j,\left|P_{i j}\right|$. The nodes are then grouped using constrained spectral clustering, which will be described in this section, to solve the optimization problem described in (8).

In Fig. 2, a flowchart depicting the execution of the SCCI algorithm is presented. In the text below a detailed description of both algorithm steps is presented.

\section{A. Step 1: Determining Coherent Generator Groups}

The coherent generator groups in the power system being considered can be found by constructing a graph that represents the dynamic coupling between the generator nodes, referred to as a dynamic graph. Normalized spectral clustering is then applied to this graph to cluster the generator nodes based on their dynamic coupling.

A dynamic graph $\mathbf{G}_{\mathbf{D}}\left(\mathbf{V}_{\mathbf{G}}, \mathbf{E}_{\mathbf{D}}, \mathbf{W}_{\mathbf{D}}\right)$ can be constructed for the $m$ generator nodes by defining its Laplacian matrix $\mathbf{L}_{\mathbf{D}}$ as [21]

$$
\left[\mathbf{L}_{\mathbf{D}}\right]_{i j}= \begin{cases}\frac{\partial P_{i j}}{\partial \delta_{i j}}=-\left|V_{i}\right|\left|V_{j}\right| B_{i j}^{\prime} \cos \left(\delta_{i}-\delta_{j}\right) & \text { if } i \neq j \\ -\sum_{l=1, l \neq i}^{m}\left[\mathbf{L}_{\mathbf{D}}\right]_{i l} & \text { if } i=j\end{cases}
$$

where $B_{i j}^{\prime}$ is the imaginary entry of the network admittance matrix, reduced to the internal generator nodes [18]. The dynamic graph $\mathbf{G}_{\mathbf{D}}$ describes the dynamic coupling between generator 
nodes; its edge weights are defined as the synchronizing coefficient $\partial P_{i j} / \partial \delta_{i j}$.

Using the Laplacian Matrix $\mathbf{L}_{\mathbf{D}}$, the linearized second-order dynamic model of the $m$-generator power system can be rewritten as [18]

$$
\mathbf{M} \ddot{\mathbf{x}}=\mathbf{L}_{\mathbf{D}} \mathbf{x}
$$

where $\mathbf{M}=\operatorname{diag}\left(2 H_{1} / \omega_{0}, 2 H_{2} / \omega_{0}, \ldots, 2 H_{m} / \omega_{0}\right)$ and is the inertia matrix.

From (4) and (10), it can be observed that (4) is a kind of Ncut of the dynamic graph $\mathbf{G}_{\mathbf{D}}$, where the graph is normalized by node inertia rather than node weight. By applying normalized spectral clustering to the dynamic graph $\mathbf{G}_{\mathbf{D}}$, the minimum Ncut of $\mathbf{G}_{\mathbf{D}}$, i.e., the solution of the optimization problem (5), can be found [17].

The first step of the SCCI algorithm can thus be executed as follows:

1) Construct the dynamic graph $\mathbf{G}_{\mathbf{D}}$ using only generator nodes, and with edge weights equal to $\partial P_{i j} / \partial \delta_{i j}$.

2) Compute the first two eigenvectors $\vartheta_{1}, \vartheta_{2}$ of the generalized eigen-problem $\mathbf{L}_{\mathbf{D}} \vartheta=\lambda \mathbf{M} \vartheta$.

3) Let $\mathbf{J} \in \mathbf{R}^{n \times 2}$ be the matrix containing the vectors $\vartheta_{1}, \vartheta_{2}$ as columns. Let $y_{i} \in \mathbf{R}^{2}$ be the vector corresponding to the $i$ th row of the matrix $\mathbf{J}$.

4) The nodes $y_{i} \in \mathbf{R}^{2}$ are then clustered into sub-sets $\mathbf{V}_{\mathbf{G} \mathbf{1}}$ and $\mathbf{V}_{\mathrm{G} 2}$ using the k-medoids algorithm.

5) Select $\mathbf{V}_{\mathbf{G} 1}$ or $\mathbf{V}_{\mathrm{G} 2}$ as the node set of a new dynamic graph and return to 1) to allow recursive bisection.

The first step of the SCCI algorithm is equivalent to the application of methods based on the theory of slow coherency. Slow coherency based methods group generators using the eigenvectors of the state matrix $\mathbf{A}$. If the inertia matrix $\mathbf{M}$ is invertible, then (2) and (12) are actually identical, assuming that $\mathbf{A}=\mathbf{M}^{-1} \mathbf{L}_{\mathbf{D}}$.

The slow coherency method is useful for offline analysis, but has some drawbacks if implemented in online applications. It is difficult to determine if the generators will retain coherency, or which oscillatory mode is excited by the disturbance that has occurred.

However, how to combine and satisfy the constraints necessary to enforce the coherent generator groups during islanding, rather than how to identify the coherent generators, is the key challenge addressed in this paper. The drawbacks of slow coherency can be overcome by using a revised slow coherency algorithm [2] or online coherency identification algorithms [23], [24].

\section{B. Step 2: Minimizing the Power-Flow Disruption While Preserving Coherent Generator Groups}

The islanding solution that will separate the coherent generator groups found in Step 1, with the minimum power-flow disruption, can be found by applying constrained spectral clustering to a static graph of the power system.

This static graph $\mathbf{G}_{\mathbf{S}}\left(\mathbf{V}, \mathbf{E}_{\mathbf{S}}, \mathbf{W}_{\mathbf{S}}\right)$ can be constructed for an $n$-node power system using power flow data to describe the active power exchange between each of the $n$ nodes.

The issue of losses within the system must be accounted for to produce the symmetric undirected graph necessary for the application of spectral clustering. To ensure that the matrix $\mathbf{W}_{\mathbf{S}}$ is symmetric, the elements of $\mathbf{W}_{\mathbf{S}}$ is defined as $\left(\left|P_{i j}\right|+\left|P_{j i}\right|\right) / 2$.
The Laplacian Matrix $\mathbf{L}_{\mathbf{S}}$ of the static graph $\mathbf{G}_{\mathbf{S}}$ can then be expressed as [21]

$$
\left[\mathbf{L}_{\mathbf{S}}\right]_{i j}= \begin{cases}\frac{\left|P_{i j}\right|+\left|P_{j i}\right|}{2}=-\left|V_{i}\right|\left|V_{j}\right| B_{i j} \sin \left(\delta_{i}-\delta_{j}\right) & \text { if } i \neq j \\ -\sum_{l=1, l \neq i}^{n}\left[\mathbf{L}_{\mathbf{S}}\right]_{i l} & \text { if } i=j\end{cases}
$$

where $B_{i j}$ is the imaginary entry of the network admittance matrix.

The minimal power-flow disruption of the graph $\mathbf{G}_{\mathbf{S}}$ is the solution of (7). However, to solve (8), the generator coherency constraint must be included. This is done by including the generator groups, obtained in the first step, as pair-wise constraints in the second step of the algorithm [26].

The pair-wise generator coherency constraints consist of 1) Must-Link constraints and 2) Cannot-Link constraints; these are defined as follows:

1) Must-Link constraints: all the generator nodes within a first-step group must be linked at the second step.

2) Cannot-Link constraints: any two generator nodes in different first-step groups cannot be linked at the second step.

Constrained spectral clustering is an efficient method for solving clustering problems with pair-wise constraints. The pair-wise constraints can be included by modifying the solution subspace using a projection matrix (the subspace approach) [26].

Without loss of generality, it can be assumed that the first $m_{1}$ nodes belong to the cluster $c_{1}$ and the next $m_{2}$ nodes belong to the cluster $c_{2}$. The projection matrix $\mathbf{U}$ can then be defined as follows [26]:

$$
\mathbf{U}=\left(\begin{array}{ccc}
\mathbf{1}_{m_{1}} & \mathbf{1}_{m_{1}} & \mathbf{0}_{m_{1} \times(n-m)} \\
\mathbf{1}_{m_{2}} & -\mathbf{1}_{m_{2}} & \mathbf{0}_{m_{2} \times(n-m)} \\
\mathbf{1}_{n-m} & \mathbf{0}_{n-m} & \mathbf{I}_{(n-m) \times(n-m)}
\end{array}\right)
$$

where $\mathbf{I}$ is the identity matrix, $\mathbf{1}$ is the all-ones column vector, and $\mathbf{0}$ is the zero matrix or zero column vector.

In this way, the solution subspace is projected from an $n$-dimension space to an $(n-m+2)$-dimension space, where $(m=$ $\left.m_{1}+m_{2}\right)$. All nodes of the same cluster in the $n$-dimension space are represented by one equivalent node in the $(n-m+$ 2)-dimension space to satisfy the pair-wise constraints.

With the introduction of the projection matrix $\mathbf{U}$, constrained spectral clustering can be applied to the static graph $\mathbf{G}_{\mathbf{S}}$ to find the cutset with minimal power-flow disruption that satisfies the generator grouping constraints produced in the first step of the SCCI algorithm. The second step of the SCCI algorithm can thus be described as follows:

1) Construct a static graph $\mathbf{G}_{\mathbf{S}}$ of all nodes with the edge weights defined as $\left(\left|P_{i j}\right|+\left|P_{j i}\right|\right) / 2$.

2) Construct the projection matrix $\mathbf{U}$ based on the generator grouping results.

3) Compute the first two eigenvectors $\vartheta_{1}, \vartheta_{2}$ of the generalized eigen-problem $\mathbf{U}^{\mathbf{T}} \mathbf{L}_{\mathbf{S}} \mathbf{U} \vartheta=\lambda \mathbf{U}^{\mathbf{T}} \mathbf{U} \vartheta$.

4) Let $\mathbf{J} \in \mathbf{R}^{n \times 2}$ be the matrix containing the vectors $\mathbf{U} \vartheta_{1}$, $\mathbf{U} \vartheta_{2}$ as columns. Let $y_{i} \in \mathbf{R}^{2}$ be the vector corresponding to the $i$ th row of $\mathbf{J}$.

5) Cluster the nodes $y_{i} \in \mathbf{R}^{2}$ into the clusters $\mathbf{V}_{\mathbf{1}}, \mathbf{V}_{\mathbf{2}}$ using the $k$-medoids algorithm. 
6) Select $\mathbf{V}_{\mathbf{1}}$ or $\mathbf{V}_{\mathbf{2}}$ as the node set of a new static graph and return to 1 ).

Using the two-step SCCI algorithm, described above, the solution of the optimization problem (8), i.e., the islanding solution, can be found.

The second step of SCCI algorithm could be used with any online coherency identification method, provided that coherent generator groups are available to serve as constraints.

\section{SCCI Algorithm TESTING}

In this Section, three test systems are used to validate the proposed SCCI algorithm [27]:

I) IEEE 9-bus test system

II) IEEE 39-bus test system

III) IEEE 118-bus test system [28].

For every test system, the SCCI algorithm has been applied and an islanding solution found. These solutions are compared with those obtained using the spectral $k$-way partitioning algorithm (SkP) and an OBDD method to evaluate the quality of the SCCI algorithm solution.

1) Spectral k-Way Partitioning (SkP): The $\mathbf{S k P}$ method introduced in [12] does not consider the generator coherency constraint; a comparison with this method in Test Case I is used to demonstrate that the generator coherency constraint is necessary to form stable islands.

SkP is a special case of the un-normalized spectral clustering algorithm described in Section II-C1. In the third step of the algorithm, instead of using $k$-medoids, to cluster the nodes $k$ reference nodes are selected and then the remaining nodes are clustered to these reference nodes according to the distance between the node and the reference nodes.

2) Ordered Binary Decision Diagram (OBDD): OBDD are capable of searching the entire searching space and finding all possible solutions [1]. Therefore, the comparisons with this method in Test Case II and Test Case III are used to demonstrate that the SCCI algorithm is capable of finding the optimal solution of (8). The OBDD method described in [1], [3], and [11] is revised to find the islanding solution with the minimal power-flow disruption:

$$
\begin{aligned}
\Phi= & S S C \cdot M C C \cdot M P D \\
S S C= & \left(\prod_{\forall i \in \mathbf{V}_{\mathbf{G} 1}, \forall j \in \mathbf{V}_{\mathbf{G} 2}} \bar{\Lambda}_{i j}^{\star}\right) \\
& \cdot\left(\prod_{k=1}^{2}\left(\prod_{\forall i, j \in \mathbf{V}_{\mathbf{G} k}} \boldsymbol{\Lambda}_{i j}^{\star}\right)\right) \\
& \cdot\left(\prod_{\forall i \in \mathbf{V}_{\mathbf{L}}}\left(\sum_{k=1}^{2} \sum_{j \in \mathbf{V}_{\mathbf{G} \mathbf{k}}}\left(\boldsymbol{\Lambda}_{i j}^{\star}\right)\right)\right) \\
M C C= & \prod_{i, j \in \mathbf{V}}\left(\boldsymbol{\Lambda}_{i j} \cdot \boldsymbol{\Lambda}_{i j}^{\star}+\overline{\boldsymbol{\Lambda}}_{i j} \cdot \overline{\boldsymbol{\Lambda}}_{i j}^{\star}\right) \\
M P D= & \left\langle\left(\sum_{i, j \in \mathbf{V}} \overline{\boldsymbol{\Lambda}}_{i j} \cdot\left|w_{i j}\right|\right)<\varepsilon\right\rangle
\end{aligned}
$$

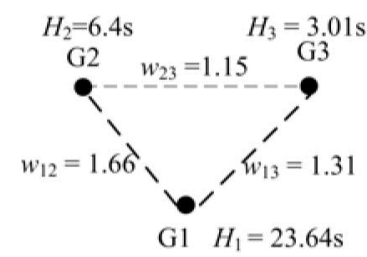

(a)

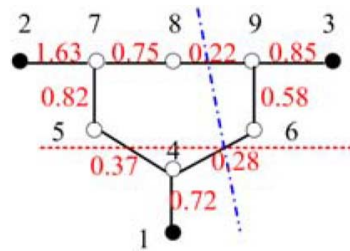

(b)
Fig. 3. Dynamic graph and static graph of the IEEE 9-bus test system (base power $S_{N}=100 \mathrm{MW}$ ). Right: the dotted line is the SCCI solution, while the dash-dotted line is the SkP solution. (a) Dynamic graph. (b) Static graph.

where $\Phi$ is the Boolean function of the OBDD method, $\boldsymbol{\Lambda}$ and $\Lambda^{\star}$ represent the adjacency matrix and reachability matrix of graph $\mathbf{G}_{\mathbf{S}}$, respectively, and $\mathbf{V}_{\mathbf{L}}$ represents the load node set of graph $\mathbf{G}_{\mathbf{S}}[1]$.

The SSC represents the requirement of generator coherency, where the first item denotes that the generators of different coherent groups cannot be connected, the second item denotes that the generators in the same coherent group must be connected, and the third item denotes that every load must be connected to one and only one coherent generator group [1], [3], [11].

The MCC is used to reduce the number of islanding solutions by allowing only those edges that help to form islands to be disconnected [11]. The MPD denotes that the power-flow disruption cannot exceed the threshold $\varepsilon$, and it is used to replace the power balance constraint (PBC) in [3] and [11].

\section{A. Evaluating Solution Quality}

1) Test Case I: IEEE 9-Bus Test System: The first step in applying the SCCI algorithm to the IEEE 9-bus test system was to construct the dynamic graph using (11), as shown in Fig. 3(a). The normalized spectral clustering algorithm, described in Section III-A, was then applied and two coherent generator groups $\{1\}$ and $\{2,3\}$ were found.

The second step of the SCCI algorithm required a static graph to be constructed using (13), as shown in Fig. 3(b). The coherent generator groups are then used to construct a projection matrix (14) that represented the must-link constraint between generators 2 and 3, and the cannot-link constraint between generator 1 and generators 2 and 3 . This projection matrix allows the constrained spectral clustering algorithm, described in Section III-B, to be used to cluster the nodes of the system.

Applying the SCCI algorithm to the IEEE 9-bus test system resulted in finding a single cutset that created an islanding solution that consisted of two islands $\{1,4\}$ and $\{2,3,5,6,7,8,9\}$. This cutset is marked in Fig. 3(b) by a dotted line, the cut of which is 0.65 p.u.

Application of the SkP method to the IEEE 9-bus test system resulted in an islanding solution that consisted of two different islands $\{3,6,9\}$ and $\{1,2,4,5,7,8\}$. This cutset is marked in Fig. 3(b) by a dash-dotted line, the cut of which is 0.50 p.u.

It is clear that the island $\{1,2,4,5,7,8\}$ is not stable because it contains the unsynchronized generators 1 and 2 . The solution of the SCCI algorithm has a higher cut; this difference from the solution of the SkP method represents the cost of satisfying the generator coherency constraint. 
TABLE I

Generator Groups IN the First Step of 39-Bus Test System

\begin{tabular}{ccc}
\hline Group 1 & Group 2 & Group 3 \\
\hline G2,G3,G4,G5,G6,G7 & G8,G9,G10 & G1 \\
\hline
\end{tabular}

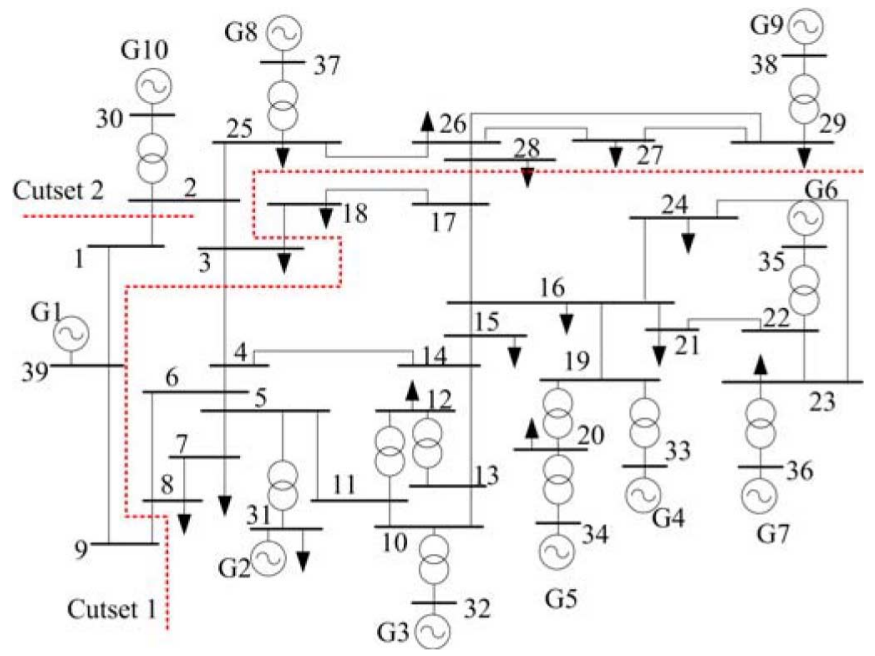

Fig. 4. Single-line diagram of the IEEE 39-bus test system. The dotted lines represent the two cutsets necessary to produce the final islanding solution.

TABLE II

The 39-Bus Test System Results Comparison With OBDD

\begin{tabular}{lcc}
\hline \multicolumn{1}{c}{ Cutset 1 } & $\sum / P_{i j /}(M W)$ & Method \\
\hline $\mathbf{( 1 )} \mathbf{8 - 9}, \mathbf{3 - 4}, \mathbf{3 - 1 8}, \mathbf{1 7 - 2 7}$ & $\mathbf{1 7 5 . 3}$ & SCCI \\
\hline $\mathbf{( 1 )} \mathbf{8 - 9 , 3 - 4 , ~ 3 - 1 8 , 1 7 - 2 7}$ & $\mathbf{1 7 5 . 3}$ & \\
(2) 9-39, 3-4, 3-18, 17-27 & 179.0 & \\
(3) $8-9,3-4, \mathbf{1 7 - 1 8}, 17-27$ & 331.2 & OBDD \\
(4) 8-9, 3-4, 16-17 & 334.3 & \\
(5) $9-39,3-4,17-18,17-27$ & 335.0 & \\
\hline
\end{tabular}

2) Test Case II: IEEE 39-Bus Test System: As in Test Case I, the first step of the SCCI algorithm was applied to find the dynamic graph of the IEEE 39-bus test system. The application of normalized spectral clustering identified the set of generator groups shown in Table I. From these groups, a set of pair-wise generator constraints can be determined to form the projection matrix.

The execution of the second step of the SCCI algorithm is effected by there being three generator groups. This effect is that the second step of the algorithm now requires the use of recursive bisection to determine two cutsets and create three islands.

The first cutset to be found, Cutset 1, separated Group 1 from Group 2 and Group 3. The second cutset to be found, Cutset 2, separated Group 3 from Group 2. Combined, these two cutsets form the final islanding solution marked in Fig. 4.

The OBDD method was executed with a $\varepsilon$ value of $335 \mathrm{MW}$ and the five solutions for Cutset 1 that had the smallest cuts are shown in Table II, alongside the SCCI algorithm solution for Cutset 1. It is obvious that the SCCI algorithm found the minimum cut for separating Group 1 from Group 2 and Group 3. A comparison of Cutset 2 is not included because it would be quite trivial for separating only one generator.
TABLE III

GeNerator Groups of 118-Bus Test System

\begin{tabular}{ccc}
\hline Group 1 & Group 2 & Group 3 \\
\hline $10,12,25,26,31$ & $46,49,54,59,61,65$, & $87,89,100,103,111$ \\
\hline
\end{tabular}

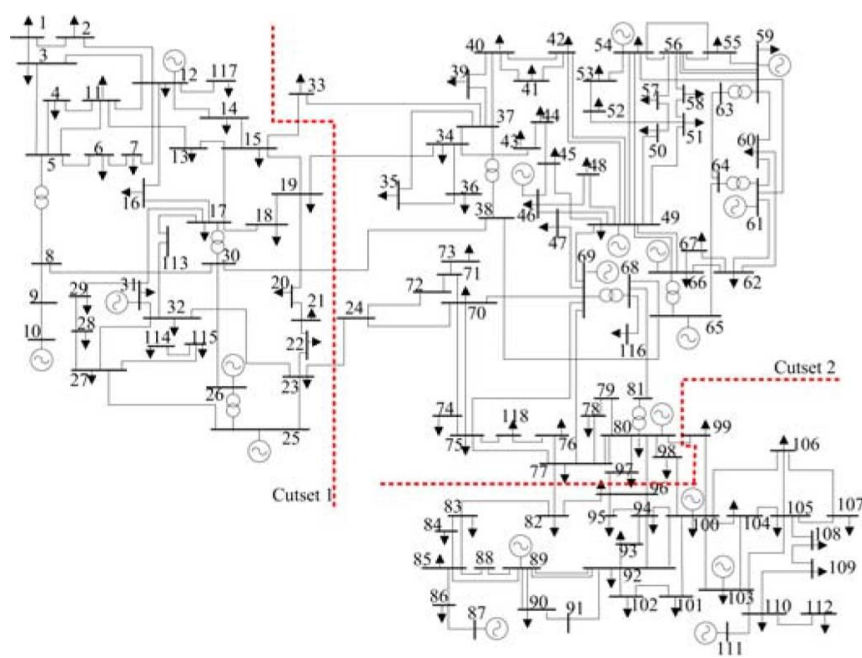

Fig. 5. Single-line diagram of the IEEE 118-bus power network. The two dotted lines represent the cutsets necessary to produce the final islanding solution.

3) Test Case III: IEEE 118-Bus Power System: The first step of the SCCI algorithm returned the three coherent generator groups given in Table III.

The two cutsets produced in the second step of the SCCI algorithm, Cutset 1 and Cutset 2, separated Group 1 from Groups 2 and 3, and then separated Group 2 from Group 3, respectively. Combined, these two cutsets form the final islanding solution marked in Fig. 5.

As in Test Case II, the results returned by the OBDD method will be used to validate the SCCI solution. Unfortunately, it is not practical to apply the OBDD method directly to the 118node network. The original network is simplified to a 34-node and 43-edge graph when searching for Cutset 1 , and is simplified to a 24-node and 38-edge graph when searching for Cutset 2 . The cutset solutions found for these simplified graphs were then mapped onto the original graphs so that all possible solutions in the original graphs are still found. The precise nature of the simplified graphs and the mapping relationships are given in the Appendix.

For both Cutset 1 and Cutset 2 the five cutsets with the smallest cut that were found by the OBBD method are presented in Table IV, alongside which are the results returned by the SCCI algorithm.

The comparison in Table IV shows that, as in Test Case II, the SCCI algorithm returned the cutset that separated the coherent generator groups with minimum cut.

\section{B. Computational Efficiency}

The accuracy of the islanding solution produced is not the sole measure of a controlled islanding algorithm performance. The computational efficiency of the algorithm is also a key 
TABLE IV

THE 118-Bus System RESUlts COMPARISON WITH OBDD

\begin{tabular}{|c|c|c|}
\hline Cutset 1 & $\begin{array}{l}\Sigma / P_{i j} / \\
(M W)\end{array}$ & Method \\
\hline (1) $15-33,19-34,30-38,23-24$ & 71.6 & SCCI \\
\hline (1) $15-33,19-34,30-38,23-24$ & 71.6 & \\
\hline (2) $15-33,19-34,30-38,24-70,24-72$ & 73.5 & \\
\hline (3) $33-37,19-34,30-38,23-24$ & 76.3 & OBDD \\
\hline (4) $33-37,19-34,30-38,24-70,24-72$ & 78.1 & \\
\hline (5) $15-33,19-34,30-38,24-70,71-72$ & 83.2 & \\
\hline Cutset 2 & $\begin{array}{l}\Sigma / P_{i j} / \\
(M W)\end{array}$ & Method \\
\hline (1) 77-82, 96-97, 80-96, 98-100, 80-99 & 52.7 & SCCI \\
\hline (1) 77-82, 96-97, 80-96, 98-100, 80-99 & 52.7 & \\
\hline (2) $77-82,96-97,80-96,98-100,99-100$ & 57.3 & \\
\hline (3) $77-82,82-96,94-96,95-96,98-100,80-99$ & 65.1 & OBDD \\
\hline (4) $77-82,80-97,80-96,98-100,80-99$ & 65.9 & \\
\hline (5) $77-82,82-96,94-96,95-96,98-100,99-100$ & 69.6 & \\
\hline
\end{tabular}

TABLE V

Computation Time of Test CASES

\begin{tabular}{ccc}
\hline Case & Method & Time $^{a}(s)$ \\
\hline 39-bus & SCCI & $\approx 0.004$ \\
118-bus & SCCI & $\approx 0.11$ \\
\hline a: Pentium 2.4GHz; 4G RAM PC; Matlab 7.0 code. &
\end{tabular}

index when evaluating the performance of a controlled islanding algorithm.

Using the minimal power-flow disruption as the objective function means that the problem the SCCI algorithm solves is a P-problem because it can be converted into a max-flow/min-cut problem and solved efficiently [9], [16].

However, the introduction of the pair-wise constraints, especially Cannot-Link constraints, necessary to include the generator coherency constraint into the problem increases its complexity. This increase in complexity makes the feasibility problem of constrained spectral clustering NP-complete in several situations, this means in polynomial time it is not possible to identify if a solution that satisfies all constraints even exists [29].

This increase in complexity can be overcome by using recursive bisection to identify the islands. This is because this type of problem can always be solved efficiently when the number of clusters is two [29].

The search space for the controlled islanding problem solved by the SCCI algorithm will be $2^{d}$ for a graph with $m$ generators, $n$ nodes, and $d$ edges. The major computational task in spectral clustering is computing the eigenvectors of the Laplacian matrix. So, the time complexity of the first step of the SCCI algorithm is just $O\left(\mathrm{~m}^{3}\right)$, and the time complexity of the second step of the SCCI algorithm is $O\left((n-m+2)^{3}\right)$. If deemed necessary, this could be reduced to $O\left((n-m+2)^{4 / 3}\right)$ as the $\mathbf{L}_{\mathbf{S}}$ matrix is a sparse matrix [15].

This degree of time complexity means that the SCCI algorithm is computationally efficient. The computational times for Test Case II and III are shown in Table V.

As mentioned in Section I, solving the problem of controlled islanding for a minimal power imbalance objective function is an NP-hard problem, a type of problem that cannot be solved in

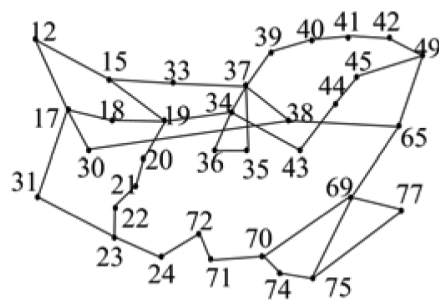

(a)

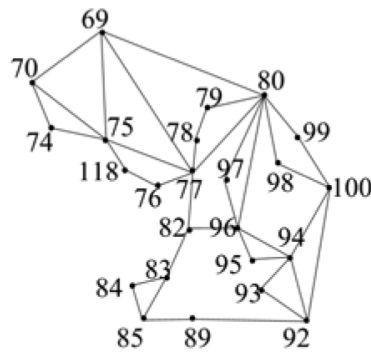

(b)
Fig. 6. Simplified graphs of 118-bus system for OBDD. (a) Simplified 34-node, 43-edge graph for Cutset 1. (b) Simplified 24-node, 38-edge graph for Cutset 2.

polynomial time, with existing algorithms, as the computational time is exponential in order [9], [11]. Thus, using the minimal power-flow disruption has the benefit of reducing the time complexity of the problem from NP-hard to P, and making the proposed SCCI algorithm computationally efficient.

\section{CONCLUSION}

This paper proposes a novel two-step SCCI algorithm for determining islanding solutions for power systems. At the core of this algorithm is a single optimization problem that uses the minimal power-flow disruption as objective function and considers ensuring generator coherency as a constraint.

Using the minimal power-flow disruption as the objective function, instead of the minimal power imbalance, improves the transient stability of the islands produced, reduces the time complexity of the problem and allows a computationally efficient algorithm to be developed. The inclusion of the generator coherency constraints prevents islands from being formed that contain non-coherent generators or isolated loads. Three test cases have been considered to evaluate the algorithm. The results show that the novel SCCI algorithm is computationally efficient and is suitable for use as a real time application, particularly in large power systems.

\section{APPENDIX}

This appendix contains the simplified graphs, Fig. 6, used in Test Case III (Section IV-A3) and the mapping relationship between these simplified graphs and the original graph, Tables VI and VII.

The simplified graphs were created with the intent of preserving the network structure between the two coherent generator groups as much as possible, while reducing the remaining network as much as possible.

If a new edge in Tables VI and VII has several mapping cutsets in the original graph, only the one with minimum weight is selected as the new edge, the others will be considered when mapping the solution found back onto the original graph so that all of the possible solutions will still be found.

For example, the new edge 12-17 in Table VI has two mapping cutsets $(15-17,12-16)$ and $(15-17,16-17)$ with the weight 94.8 and 105.0, respectively. The weight of the new edge $12-17$ will be designated as 94.8 . If the new edge $12-17$ 
TABLE VI

MAPPING Nodes AND EDGES OF SimPLifIEd GRAPH 1

\begin{tabular}{clcc}
\hline New node & Original nodes & New node & Original nodes \\
\hline 12 & $1-14,16,117$ & 49 & $46-58$ \\
31 & $25-29,31-32,113-115$ & 71 & 71,73 \\
65 & $59-68,78-112,116$ & 77 & $76-77,118$ \\
\hline New edge & Original cutsets & New edge & Original cutsets \\
\hline $23-31$ & $(23-32,23-25)$ & $24-72$ & $(24-70,24-72)$ \\
$71-72$ & $(24-70,71-72)$ & $70-71$ & $(24-70,70-71)$ \\
$37-39$ & $(37-39,37-40)$ & $39-40$ & $(39-40,37-40)$ \\
$40-41$ & $(40-41,40-42)$ & $41-42$ & $(41-42,40-42)$ \\
$45-49$ & $(45-46,45-49)$ & $49-65$ & $(49-66,47-69,49-69)$ \\
$70-74$ & $(70-74,70-75)$ & $74-75$ & $(70-75,74-75)$ \\
\hline New edge & \multicolumn{3}{c}{ Original cutsets } \\
$12-15$ & $(15-17,14-15,13-15) ;(15-17,12-14,13-15)$ \\
$12-17$ & $(15-17,14-15,11-13) ;(15-17,12-14,11-13)$ \\
$17-31$ & $(15-17,12-16) ;(15-17,16-17)$ & \\
$65-69$ & $(69-31,17-113,26-30) ;(17-31,113-32,26-30)$ \\
$69-77$ & $(69-77,68-89,49-69) ;(65-68,68-81,47-69,49-69)$ \\
$75-77$ & $(75-77,75-118) ;(75-77,76-77) ;(75-77,76-118)$ \\
\hline
\end{tabular}

TABLE VII

MAPPING NODES AND EDGES OF SIMPLIFIEd GRAPH 2

\begin{tabular}{clcc}
\hline New node & Original nodes & New node & Original nodes \\
\hline 69 & $1-20,33-69,81,116,117$ & 89 & $88-89$ \\
70 & $21-32,70-73,113-115$ & 92 & $90-92$ \\
85 & $85-87$ & \multicolumn{3}{c}{100} & $100-112$ \\
\hline New edge & \multicolumn{3}{c}{ Original cutsets } \\
\hline $69-80$ & $(68-69,68-65) ;(68-81) ;(80-81)$ \\
$85-89$ & $(85-88,85-89) ;(88-89,85-89)$ \\
$89-92$ & $(80-90,89-92) ;(90-91,89-92) ;(91-92,89-92)$ \\
$92-100$ & $(92-100,92-102) ;(92-100,101-102) ;(92-100,100-101)$ \\
\hline
\end{tabular}

is in the solution produced, the mapping cutsets $(15-17,12-16)$ or $(15-17,16-17)$ are possible elements of the final solution for the original graph.

\section{REFERENCES}

[1] Q. Zhao, K. Sun, D. Zheng, J. Ma, and Q. Lu, “A study of system splitting strategies for island operation of power system: A two-phase method based on OBDDs," IEEE Trans. Power Syst., vol. 18, no. 4, pp. 1556-1565, Nov. 2003.

[2] X. Wang, "Slow coherency grouping based islanding using minimal cutsets and generator coherency index tracing using the continuation method," Ph.D. dissertation, Iowa State Univ., Ames, 2005.

[3] K. Sun, D. Zheng, and Q. Lu, "Splitting strategies for islanding operation of large-scale power systems using OBDD-based methods," IEEE Trans. Power Syst., vol. 18, no. 2, pp. 912-922, May 2003.

[4] L. Liu, W. Liu, D. A. Cartes, and I.-Y. Chung, "Slow coherency and Angle Modulated Particle Swarm Optimization based islanding of large-scale power systems," Adv. Eng. Informat., vol. 23, pp. 45-56, 2009.

[5] Q. Zhao, X. Li, and D. Zheng, "OBDD-based load shedding algorithm for power systems," in Handbook Power Systems I, Energy Systems. Berlin, Germany: Springer, 2010, pp. 235-253.

[6] V. Terzija, "Adaptive underfrequency load shedding based on the magnitude of the disturbance estimation," IEEE Trans. Power Syst., vol. 21 , no. 3, pp. 1260-1266, Aug. 2006.

[7] S. Najafi, "Evaluation of interconnected power systems controlled islanding," in Proc. IEEE Bucharest Power Tech Conf., Bucharest, Romania, 2009.

[8] C. Wang, B. Zhang, Z. Hao, J. Shu, P. Li, and Z. Bo, "A novel real-time searching method for power system splitting boundary," IEEE Trans. Power Syst., vol. 25, no. 4, pp. 1902-1909, Nov. 2010.
[9] A. Sen, P. Ghosh, V. Vittal, and B. Yang, "A new min-cut problem with application to electric power network partitioning," Eur. Trans. Elect. Power, vol. 19, no. 6, pp. 778-797, 2009.

[10] C. H. Papadimitriou, Computational Complexity. Reading, MA: Addison-Wesley, 1994.

[11] Y. Qiao, "Study on the active splitting control scheme of large-scale power systems," Ph.D. dissertation, Tsinghua Univ., Beijing, China, 2007.

[12] L. Hao, G. W. Rosenwald, J. Jung, and C. C. Liu, "Strategic power infrastructure defense," Proc. IEEE, vol. 93, no. 5, pp. 918-933, May 2005.

[13] A. Peiravi and R. Ildarabadi, "A fast algorithm for intentional islanding of power systems using the multilevel kernel k-means approach," $J$. Appl. Sci., vol. 9, no. 12, pp. 2247-2255, 2009.

[14] B. Yang, V. Vittal, G. T. Heydt, and A. Sen, "A novel slow coherency based graph theoretic islanding strategy," in Proc. IEEE PES General Meeting, Tampa, FL, 2007.

[15] A. Peiravi and R. Ildarabadi, "Comparison of computational requirements for spectral and kernel k-means bisection of power system," Australian J. Basic Appl. Sci., vol. 3, no. 3, pp. 2366-2388, 2009.

[16] U. V. Luxburg, "A tutorial on spectral clustering," Statist. Comput., vol. 17, no. 4, pp. 395-416, Dec. 2007.

[17] J. Shi and J. Malik, "Normalized cuts and image segmentation," IEEE Trans. Pattern Anal. Mach. Intell., vol. 22, no. 8, pp. 888-905, Aug. 2000.

[18] J. H. Chow, Time-Scale Modeling of Dynamic Networks With Applications to Power Systems. New York: Springer- Verlag, 1982.

[19] S. S. Lamba and R. Nath, "Coherency identification by the method of weak coupling," Elect. Power Energy Syst., vol. 7, no. 4, pp. 233-242, Oct. 1985.

[20] V. E. Henner, "A network separation scheme for emergency control," Int. J. Elect. Power Energy Syst., vol. 2, no. 2, pp. 109-114, 1980.

[21] L. Ding and V. Terzija, "A new controlled islanding algorithm based on spectral clustering," in Proc. 4th IEEE Int. Conf. Electric Utility Deregulation, Restructuring, and Power Technology (DRPT2011), Weihai, China, Jul. 2011.

[22] [Online]. Available: http://en.wikipedia.org/wiki/Arg_max.

[23] S. Theodoridis and K. Koutroumba, Pattern Recognition, 4th ed. New York: Academic, 2008.

[24] M. Johnson, M. Begovic, and J. Daalder, "A new method suitable for real-time generator coherency determination," IEEE Trans. Power Syst., vol. 19, no. 3, pp. 1473-1482, Aug. 2004.

[25] N. Senroy, "Generator coherency using the hilbert-huang transform," IEEE Trans. Power. Syst., vol. 23, no. 4, pp. 1701-1708, Nov. 2008.

[26] T. D. Bie, J. Suykens, and B. D. Moor, "Learning from general label constraints," in Proc. IAPR Int. Workshop Statistical Pattern Recognition, Lisbon, Portugal, Aug. 2004.

[27] [Online]. Available: http://www.ee.washington.edu/research/pstca/.

[28] K. Sun, D. Zheng, and Q. Lu, "A simulation study of OBDD-based proper splitting strategies for power systems under consideration of transient stability," IEEE Trans. Power Syst., vol. 20, no. 1, pp. 389-399, Feb. 2005

[29] I. Davidson and S. S. Ravi, "The complexity of non-hierarchical clustering with instance and cluster level constraints," Data Mining Knowl. Discov., vol. 14, no. 1, pp. 25-61, 2007.

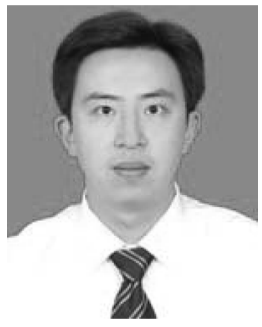

Lei Ding (M'10) was born in Shandong, China, in 1980. He received the B.E. and Ph.D. degrees in electrical engineering from Shandong University, Jinan, China, in 2001 and 2007, respectively.

From 2008 to 2009, he was a postdoctoral researcher in Tsinghua University, Beijing, China. $\mathrm{He}$ joined the School of Electrical Engineering, Shandong University as an Associate Professor in 2010. Currently he is also a Research Associate in The University of Manchester, Manchester, U.K. His research interests include controlled islanding and microgrid protection and control. 


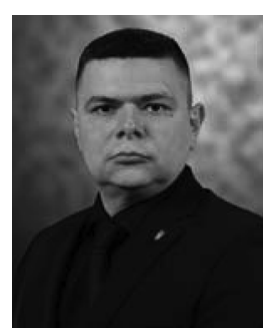

Francisco M. González-Longatt (M'03-SM'09) received his the Ph.D. degree in electrical engineering from the Universidad Central de Venezuela in 2008.

$\mathrm{He}$ is a former Associate Professor in the Electrical Engineering Department of the Universidad Nacional Politécnico de la Fuerza Armada Nacional, Venezuela (1995-2009). He is currently a Research Associate in the School of Electrical and Electronic Engineering, The University of Manchester, Manchester, U.K

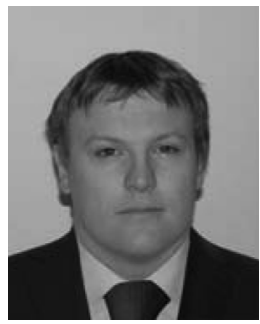

Peter Wall received the Bachelors and Masters degrees in electrical engineering from the University of Manchester, Manchester, U.K., in 2008 and 2009, respectively. He is currently pursuing the Ph.D. degree in the School of Electrical and Electronic Engineering, The University of Manchester.

His main area of interest is power system dynamics and frequency stability.

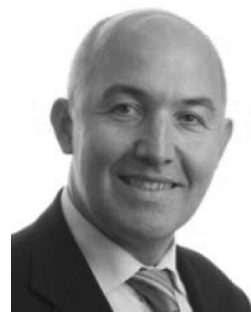

Vladimir Terzija (M'95-SM'00) is the EPSRC Chair Professor in Power System Engineering in the School of Electrical and Electronic Engineering, The University of Manchester, Manchester, U.K.

From 1997 to 1999, he was an Assistant Professor at the University of Belgrade, Belgrade, Serbia. From 2000 to 2006, he was with ABB AG, Germany, working as an expert for switchgear and distribution automation. His main research interests are application of intelligent methods to power system monitoring, control, and protection, switchgear and fast transient processes, as well as DSP applications in power systems. 